\title{
3D Microstructure Reconstruction and Characterization of Solid-State Electrolyte with Varying Porosity
}

Tanner R. Hamann ${ }^{1}$, Lei Zhang ${ }^{1}$, Yunhui Gong ${ }^{1}$, Griffin L. Godbey ${ }^{1}$, Jack E. Gritton ${ }^{1}$, Zhaohui Ma ${ }^{1}$, Dennis W. McOwen ${ }^{1}$, Gregory T. Hitz ${ }^{1}$, Liangbing Hu${ }^{1}$, Eric D. Wachsman ${ }^{1}$

${ }^{1}$ University of Maryland, College Park, MD, USA

Solid-state electrolytes are promising candidates to replace organic liquid electrolytes in lithium-ion batteries [1]. However, successfully integrating solid electrolytes with electrode materials requires overcoming the low ionic conductivity of solid electrolytes and the high interfacial impedance associated with poor electrolyte-electrode contact $[2,3]$. The garnet-like ceramic $\mathrm{Li}_{7} \mathrm{La}_{3} \mathrm{Zr}_{2} \mathrm{O}_{12}$ (LLZ) and its derivatives have displayed ionic conductivities of $10^{-4} \mathrm{~S} / \mathrm{cm}$ as well as good stability against lithium [4, 5]. Simultaneously, surface chemistry modifications of the LLZ-electrode interface have greatly improved solid-solid contact between LLZ and lithium anodes [6]. Recently, the LLZ-electrode interfacial impedance was further reduced by replacing the planar geometry with a variable-porosity multi-layered structure. This approach dramatically increased the electrolyte-electrode interfacial area, and the thickness and porosity of each layer could be adjusted to optimize battery performance. However, existing research on the relationship between non-planar garnet microstructures and overall device performance is limited.

Here, we investigated the structural and electrical properties of $\mathrm{Li}_{7} \mathrm{La}_{3} \mathrm{Zr}_{2} \mathrm{O}_{12}$ electrolyte structures with varying porosities. The LLZ material was synthesized via solid-state reaction of precursor powders. Samples with varying porosity were created via tape casting of the LLZ particles and sintering on a powder bed in tube furnaces at $1050{ }^{\circ} \mathrm{C}$ while flowing oxygen. PMMA pore former was added to the tape slurries to control the porosity in the sintered sample. Gold electrodes were painted onto the samples and annealed at $700{ }^{\circ} \mathrm{C}$ while flowing oxygen. The samples were sealed in pouch cells in argon, then placed in an environmental chamber for EIS testing at varying temperatures. Samples were removed from the pouch cells, with part of the sample ground for XRD and the remaining part imaged by SEM in SE and BSE modes to calculate average sample porosity and electrode separation. Several samples were selected for 3D FIB tomography (Figure 1, left), wherein a focused ion beam was used to deposit platinum and mill a trench around a region of interest (Figure 1, middle), and serially mill-and-image cross-sections to produce 3D image sets (Figure 1, right). The images were pre-processed (Figure 2, left), segmented into discrete phases (Figure 2, middle), and the 3D microstructures reconstructed for analysis (Figure 2, right).

3D reconstructions showed that the pore and LLZ microstructures remained continuous even at intermediate porosities of $\sim 50 \%$. The measured average z-orientation (MAZO) angle was extracted from the $3 \mathrm{D}$ reconstruction by calculating the average angle between segments of the microstructure skeleton and the z-axis (perpendicular to the surface of the porous layer) [7]. The MAZO angles of the pore and LLZ microstructures were somewhat higher than the MAZO angle expected for a randomly-oriented microstructure, indicating some structural settling during sintering. Estimates of sample porosity from 2D cross-sectional SEM images appeared to agree with the sample porosity calculated from the 3D reconstructions. SEM images of samples after EIS testing showed that the gold electrodes remained primarily at the sample surface and only minimally penetrated into the sample. Based on EIS measurements, the calculated resistance for lithium-ion transport through the bulk electrolyte was found to increase substantially as sample porosity increased. The increase in bulk resistance was also correlated to the tortuosity of the LLZ microstructure, which was calculated from the 3D reconstructions. 


\section{References:}

[1] Sun, C., et al, Nano Energy 33 (2017), p. 363.

[2] Luo, W., et al, Journal of the American Chemical Society 138 (2016), p.12258.

[3] Takada, K., et al, Acta Materialia 61 (2013), p.759.

[4] Huang, M., et al, Solid State Ionics 204-205 (2011), p.41.

[5] Murugan, R., et al, Angewandte Chemie (International ed. in English) 46 (2007), p. 7778.

[6] Han, X., et al, Nature Materials 1 (2016), p. 572.

[7] Gostovic, D., et al, Journal of the American Ceramic Society 94 (2011), p. 620.
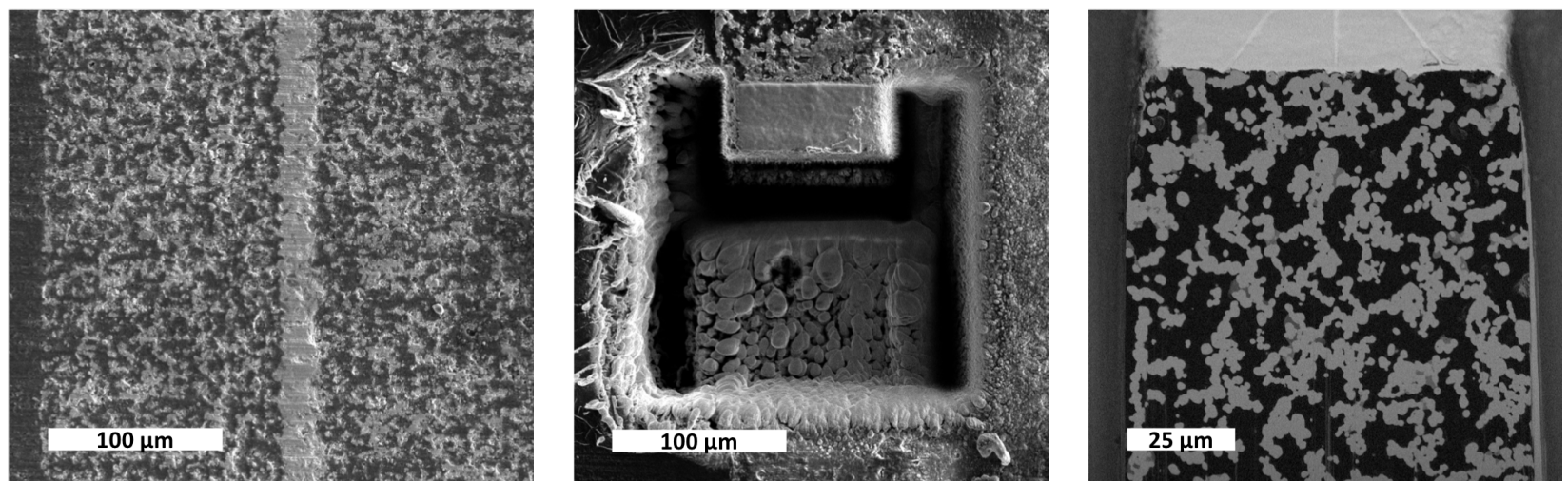

Figure 1. sample selected for 3D FIB tomography (left); platinum deposited onto and trench dug around region of interest (middle); and SEM image obtained during serial milling-and-imaging (right).
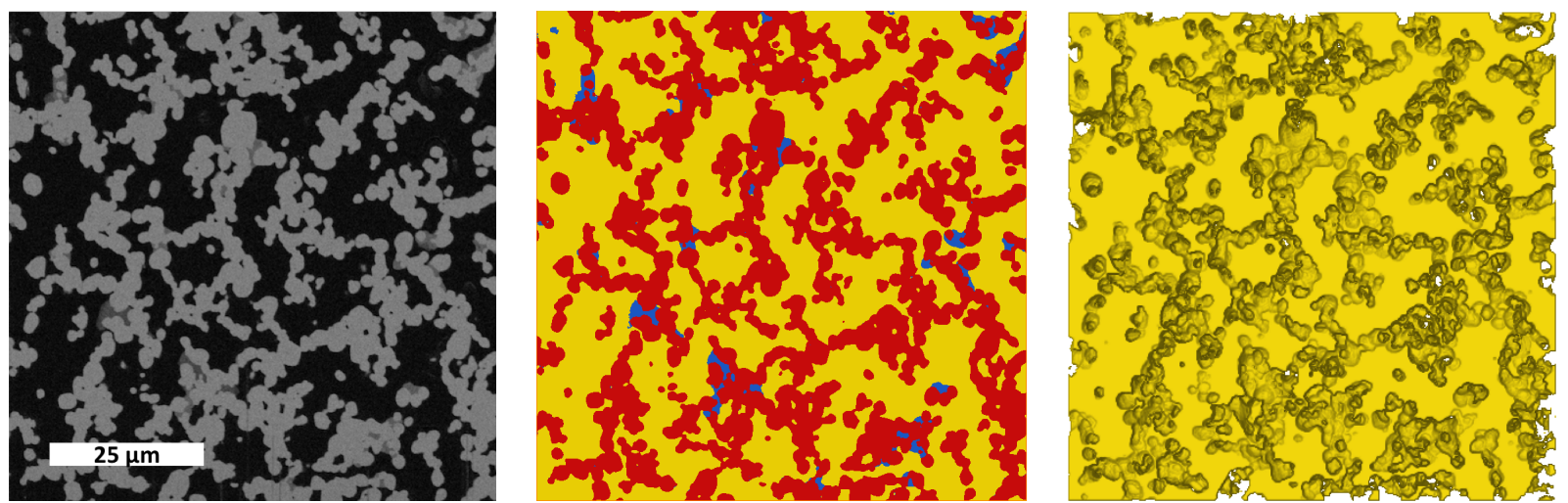

Figure 2. SEM image after pre-processing (left); image segmented into discrete phases based on pixel intensity (middle); and 3D reconstruction of pore structure from segmented image (right). 